\title{
Epidemiological trends of pancreatic and gastrointestinal neuroendocrine tumors in Japan: a nationwide survey analysis
}

\author{
Tetsuhide Ito - Hisato Igarashi - Kazuhiko Nakamura - Hironobu Sasano • \\ Takuji Okusaka $\cdot$ Koji Takano $\cdot$ Izumi Komoto $\cdot$ Masao Tanaka • \\ Masayuki Imamura $\cdot$ Robert T. Jensen $\cdot$ Ryoichi Takayanagi $\cdot$ Akira Shimatsu
}

Received: 29 July 2013/Accepted: 10 January 2014/Published online: 6 February 2014

(C) Springer Japan 2014

\begin{abstract}
Background Although neuroendocrine tumors (NETs) are rare, the number of patients with NET is increasing. However, in Japan, there have been no epidemiological studies on NET since 2005; thus, the prevalence of NET remains unknown.

Methods We reported the epidemiology of gastroenteropancreatic neuroendocrine tumors (GEP-NETs) [pancreatic neuroendocrine tumors (PNETs) and gastrointestinal neuroendocrine tumors (GI-NETs)] in Japan in 2005. Here, we conducted the second nationwide survey on patients with GEP-NETs who received treatment in 2010.

Results A total of 3,379 patients received treatment for PNETs in 2010, representing a 1.2-fold increase in the number of patients from 2005 to 2010 . The prevalence was estimated to be $2.69 / 100,000$, with an annual onset incidence of 1.27/100,000 in 2010. Non-functioning tumor (NF)-PNETs comprised $65.5 \%$ of cases followed by insulinoma (20.9 \%) and gastrinoma (8.2\%). Interestingly, the number of patients with NF-PNETs increased $\sim 1.8$
\end{abstract}

T. Ito $(\bowtie) \cdot$ H. Igarashi $\cdot$ K. Nakamura $\cdot$ R. Takayanagi

Department of Medicine and Bioregulatory Science, Graduate

School of Medical Sciences, Kyushu University, 3-1-1 Maidashi,

Higashi-ku, Fukuoka 812-8582, Japan

e-mail: itopapa@intmed3.med.kyushu-u.ac.jp

H. Sasano

Department of Pathology, Tohoku University School

of Medicine, Sendai, Japan

T. Okusaka

Hepatobiliary and Pancreatic Oncology Division, National

Cancer Center Hospital, Tokyo, Japan

K. Takano

Department of Nephrology and Endocrinology, Faculty

of Medicine, University of Tokyo, Tokyo, Japan fold since 2005. A total of $19.9 \%$ of patients exhibited distant metastasis at initial diagnosis; $4.3 \%$ had complications with multiple endocrine neoplasia type 1 (MEN-1), and only $4.0 \%$ had NF-PNETs associated with MEN-1. Meanwhile, an estimated 8,088 patients received treatment for GI-NETs, representing a $\sim 1.8$-fold increase since 2005 . The prevalence was estimated to be $6.42 / 100,000$, with an annual onset incidence of $3.51 / 100,000$. The locations of GI-NETs varied: foregut, $26.1 \%$; midgut, $3.6 \%$; and hindgut, $70.3 \%$. Distant metastasis and complications with MEN-1 were observed in 6.0 and $0.42 \%$ at initial diagnosis, respectively. The frequency of carcinoid syndrome in patients with GI-NETs was $3.2 \%$.

Conclusion We clarified the epidemiological changes in GEP-NETs from 2005 to 2010 in Japan.

Keywords Neuroendocrine tumor - Pancreatic neuroendocrine tumor - Gastrointestinal neuroendocrine tumor $\cdot$ Nation-wide survey $\cdot$ Epidemiology

I. Komoto $\cdot$ M. Imamura

Department of Surgery, Kansai Electric Power Company

Hospital, Osaka, Japan

M. Tanaka

Department of Surgery and Oncology, Graduate School of

Medical Sciences, Kyushu University, Fukuoka, Japan

R. T. Jensen

Digestive Diseases Branch, National Institutes of Diabetes, Digestive and Kidney Diseases, National Institutes of Health, Bethesda, MD, USA

A. Shimatsu

Clinical Research Institute, National Hospital Organization Kyoto Medical Center, Kyoto, Japan 


\section{Introduction}

Neuroendocrine tumors (NETs) are generally considered rare tumors that progress slowly [1]. However, according to the surveillance, epidemiology, and end results (SEER) study, a US epidemiological database, the number of patients has been increasing; the incidence rate of the disease increased fivefold from 1.09 per 100,000 people in 1973 to 5.25 per 100,000 people in 2004 [2]. Although the reasons for this increase are unclear, the recognition of the disease and improved diagnostic technology may be partially responsible. Thus, continued accumulation and examination of data regarding the trend of the actual number of patients is necessary [3, 4].

However, in Japan, the prevalence of gastroenteropancreatic NETs (GEP-NETs) [pancreatic endocrine tumors (PNETs) and gastrointestinal neuroendocrine tumors (GINETs)] is unclear. Consequently, a nationwide epidemiological survey of patients with GEP-NET who received treatment from January 1 to December 31, 2005 was conducted in [5]; thus, the difference in the prevalence of the disease between Japan and Western nations gradually became clear. The large differences in GEP-NETs between Japan and Western nations are primarily due to differences in the presence of multiple endocrine neoplasia type 1 (MEN-1) in NF-PETs as well as the location, symptomatic status, and prevalence of malignancy in GI-NETs [5]. The present study reports the second nationwide survey on patients with GEP-NETs who received treatment in 2010. Furthermore, the epidemiological changes in these patients from 2005 to 2010 were examined.

\section{Methods}

We conducted the second nationwide survey to examine the epidemiology of GEP-NETs in Japan. The subjects were patients with GEP-NETs including PNETs and GINETs who received treatment from January 1 to December 31, 2010. Subjects were collected using a nationwide stratified random sampling method similar to that used in the first survey [5]. In brief, the departments of gastroenterology, gastroenterological surgery, endocrinology, and metabolic medicine of each hospital were listed, and stratified random sampling was used to select departments for the survey. The sampling rates for the stratum of general hospitals with $<100,100-199,200-299,300-399$, $400-499$, and $\geq 500$ beds and university hospitals were 5 , $10,20,40,80,100$, and $100 \%$, respectively. To increase the efficiency of this survey, we added some relevant departments in which many patients with GEP-NETs were expected to be treated; they were considered a special stratum and were all selected. A questionnaire was directly mailed to the heads of the 6,339 randomly selected departments at the abovementioned sampling rates. Returned questionnaires providing information about 3,366 patients including 1,273 patients with PNETs and 2,093 with GI-NETs were collected. A response rate was $20.2 \%$. The diagnosis of GEP-NETs was classified according to the WHO 2010 criteria [6]. However, mixed adenoneuroendocrine carcinoma (MANEC) and hyperplastic and preneoplastic lesions were excluded. Regarding PNETs, patients with clinical symptoms and elevated plasma hormone levels were diagnosed as having a functioning PNET. On the other hand, patients without clinical symptoms and with no elevation of plasma hormone levels were diagnosed as having a nonfunctioning tumor (NF-PNET) regardless of whether the hormone production was evaluated by immunohistochemistry or mRNA detection in the tumor tissue.

\section{Results}

Epidemiology of PNETs in Japan

\section{Epidemiology (Table 1)}

The data collected from the present survey showed the estimated total number of patients treated for PNETs in the year 2010 was 3,379 [95\% confidence interval (CI) $3,173-3,580$ ] and the overall prevalence was 2.69 per 100,000 people (95\% CI 2.29-3.08). This represents an approximately 1.2 -fold increase since 2005 . The total number of patients treated for functioning tumors was estimated to be 1,105 (95\% CI 868-1,342), and the overall prevalence was 0.88 per 100,000 people $(95 \%$ CI $0.65-1.05)$. On the other hand, the total number of patients treated for non-functioning tumors was estimated to be 2,274 (95\% CI 1,759-2,789), and the overall prevalence was 1.81 per 100,000 people (95\% CI 1.51-2.11). There were more patients with functioning PNETs than NFPNETs in 2005, while the opposite trend was observed in 2010. The incidence rates of PNETs, functioning tumors, and NF-PETs in 2010 were estimated to be 1.27 per 100,000 people (95\% CI 1.08-1.46), 0.41 per 100,000 people (95\% CI $0.32-0.48$ ), and 0.87 per 100,000 people (95\% CI 0.72-1.01), respectively. The number of newonset functioning PNETs in 2010 was similar to that in 2005; however, the number of new-onset NF-PNETs in 2010 was approximately 1.7 -fold greater than that in 2005 .

\section{Distribution of PNETs in Japan in 2010 (Table 2)}

NF-PNETs were the most common PNETs in Japan in 2010, comprising $65.5 \%$ of all PNETs. Meanwhile, functioning tumors comprised $34.5 \%$ of PNETs. The most 
Table 1 The trends of epidemiology of pancreatic neuroendocrine tumors (PNETs) from 2005 to 2010 in Japan

\begin{tabular}{|c|c|c|}
\hline & $2005^{*}$ & 2010 \\
\hline Total number of patients treated for PNET & $2,845(95 \%$ CI $2,455-3,507)$ & $3,379(95 \%$ CI 3,173-3,580) \\
\hline Functioning tumors & $1,627(95 \%$ CI $1,404-2,005)$ & $1,105(95 \%$ CI $868-1,342)$ \\
\hline Non-functioning tumors & $1,218(95 \%$ CI $1,053-1,453)$ & $2,274(95 \%$ CI $1,759-2,789)$ \\
\hline Overall prevalence of PNETs (per 100,000 population) & $2.23(95 \%$ CI $1.93-2.76)$ & 2.69 (95\% CI 2.29-3.08) \\
\hline Functioning tumors & $1.27(95 \%$ CI $1.10-1.57)$ & 0.88 (95 \% CI 0.65-1.05) \\
\hline Non-functioning tumors & 0.95 (95\% CI $0.82-1.17)$ & $1.81(95 \%$ CI $1.51-2.11)$ \\
\hline Incidence rate of PNETs (per 100,000 population) & $1.01(95 \%$ CI $0.88-1.25)$ & 1.27 (95\% CI 1.08-1.46) \\
\hline Functioning tumors & 0.5 (95\% CI $0.44-0.62)$ & $0.41(95 \%$ CI $0.32-0.48)$ \\
\hline Non-functioning tumors & $0.51(95 \%$ CI $0.88-1.25)$ & 0.87 (95 \% CI $0.72-1.01)$ \\
\hline
\end{tabular}

*Data modified from reference [5]

$95 \%$ CI $95 \%$ confidence interval

Table 2 Distribution of pancreatic neuroendocrine tumors (PNETs) in 2010

\begin{tabular}{lll}
\hline & Number of patients & Percentage $(\%)$ \\
\hline Functioning PNETs & $439 / 1,273$ & 34.5 \\
Insulinoma & $266 / 1,273$ & 20.9 \\
Gastrinoma & $104 / 1,273$ & 8.2 \\
Glucagonoma & $42 / 1,273$ & 3.2 \\
VIPoma & $8 / 1,273$ & 0.6 \\
Somatostatinoma & $4 / 1,273$ & 0.3 \\
Others & $17 / 1273$ & 1.3 \\
Non-functioning PNETs & $834 / 1,273$ & 65.5 \\
\hline
\end{tabular}

Table 3 Percentages of neuroendocrine carcinoma (NEC) among pancreatic and gastrointestinal neuroendocrine tumors in 2010

\begin{tabular}{llc}
\hline & Number of patients & Percentage $(\%)$ \\
\hline (a) Total PNETs & $95 / 1,273$ & 7.5 \\
Functioning PNETs & $14 / 439$ & 3.2 \\
Insulinoma & $5 / 266$ & 1.9 \\
Gastrinoma & $6 / 104$ & 5.8 \\
Glucagonoma & $1 / 42$ & 2.4 \\
VIPoma & $0 / 8$ & 0 \\
Somatostatinoma & $0 / 4$ & 0 \\
Others & $2 / 17$ & 11.8 \\
Non-functioning PNETs & $81 / 834$ & 9.7 \\
(b) Total GI-NETs & $130 / 2,093$ & 6.2 \\
Foregut & $93 / 737$ & 12.6 \\
Midgut & $7 / 77$ & 9.1 \\
Hindgut & $30 / 1,279$ & 2.3 \\
\hline
\end{tabular}

PNETs pancreatic neuroendocrine tumors, GI-NETs gastrointestinal neuroendocrine tumors

frequent functioning PNETs were insulinoma (20.9\%) followed by gastrinoma (8.2 \%). Glucagonoma, VIPoma, and somatostatinoma had low frequencies of 3.2, 0.6, and $0.3 \%$, respectively.
Histopathological distribution of PNETs in Japan in 2010 (Table 3a)

The histological survey was conducted according to the 2010 WHO classification. This survey comprised 2 parts: one was for NETs $(\mathrm{G} 1 / \mathrm{G} 2)$ and the other for neuroendocrine carcinoma (NEC; small-cell or large-cell type). The frequency of NECs among all PNETs was $7.5 \%$. The frequency of NECs among NF-PNETs was high at the rate of $9.7 \%$ compared with that among functioning PNETs at the rate of $3.2 \%$.

\section{Percentages of distant metastases and association of MEN-1 in PNETs (Table 4)}

Among the patients with PNETs, $19.9 \%$ exhibited distant metastases at initial diagnosis; the percentages among functioning PNETs and NF-PNETs were $16.9 \%$ and $21.3 \%$, respectively. Among functioning PNETs, gastrinoma accounted for $30.2 \%$, whereas insulinoma accounted for $9.3 \%$. With regard to the grade of WHO calcification, the percentage of distant metastases in patients with NEC at initial diagnosis was high at the rate of $46.3 \%$ compared with that in patients with NET G1/G2 at the rate of $12.9 \%$. Especially, NF-PNETs patients with NEC was the most prevalent at the rate of $51.9 \%$.

On the other hand, complications with MEN-1 accounted for $4.3 \%$ of all PNETs (4.9\% of functioning PNETs and $4.0 \%$ of NF-PNETs). The percentage of complications with MEN-1 among cases of gastrinoma was high (16.3\%) but low among cases of insulinoma $(0.8 \%)$.

Epidemiology of GI-NETs in Japan in 2010

\section{Epidemiology (Table 5)}

The present survey estimated a total of 8,088 people $(95 \%$ CI 5,669-10,507) were treated for GI-NETs in 2010. The 
total numbers of patients treated for foregut, midgut, and hindgut tumors in this group were 2,107 (95\% CI 1,189-3,028), 290 (95\% CI 271-349), and 5,690 (95\% CI 3,583-7,797), respectively. There were approximately 1.8 times as many patients in 2010 as those in 2005. The overall prevalence of GI-NETs was 6.42 per 100,000 people (95 \% CI 4.50-8.34). The overall prevalences of foregut, midgut, and hindgut tumors were 1.67 (95\% CI 0.94-2.40), 0.23 (95\% CI 0.18-0.28), and 4.52 per 100,000 people (95 \% CI 3.17-5.87), respectively. The locations of GI-NETs varied: $26.1,3.6$, and $70.3 \%$ were in the foregut, midgut, and hindgut, respectively. Similar to the survey results from 2005, the frequency of midgut NETs was very low in Japan relative to that in Western nations. Meanwhile, the incidence rate of GI-NETs in 2010 was estimated to be 3.51 per 100,000 people (95\% CI

Table 4 Percentages of distant metastases and associated MEN-1 in pancreatic neuroendocrine tumors (PNETs) in 2010

\begin{tabular}{|c|c|c|c|c|}
\hline & \multicolumn{3}{|c|}{ Distant metastases (\%) } & \multirow{2}{*}{$\begin{array}{l}\text { Associated } \\
\text { MEN-1 } \\
(\%)\end{array}$} \\
\hline & Total & NET G1/G2 & NEC & \\
\hline Total PNETs & 19.9 & 12.9 & 46.3 & 4.3 \\
\hline Functioning PNETs & 16.9 & 17.2 & 14.3 & 4.9 \\
\hline Insulinoma & 9.3 & 9.7 & 0 & 0.8 \\
\hline Gastrinoma & 30.2 & 32.4 & 10.7 & 16.3 \\
\hline Glucagonoma & 8.3 & 9.1 & 0 & 8.3 \\
\hline VIPoma & 80.0 & 80.0 & 0 & 0 \\
\hline Somatostatinoma & 100 & 100 & 0 & 0 \\
\hline Others & 25.0 & 0 & 50 & 0 \\
\hline Non-functioning PNETs & 21.3 & 12.9 & 51.9 & 4.0 \\
\hline
\end{tabular}

$M E N-1$ multiple endocrine neoplasia type 1
2.50-4.53); the incidence rates of foregut, midgut, and hindgut tumors in this group were $1.20(95 \% \mathrm{CI}$ $0.48-1.91), 0.15$ (95\% CI 0.12-0.18), and 2.12 per 100,000 people (95\% CI 1.56-2.67), respectively. Although the incidence rates of foregut and hindgut tumors clearly increased since 2005 , no change in the incidence rate of midgut tumors was observed.

\section{Histopathological distribution of GI-NETs in Japan in 2010 (Table 3b)}

The frequency of NEC among all GI-NETs was $6.2 \%$. NEC was most common among foregut NETs $(12.6 \%)$ followed by midgut NETs $(9.1 \%)$ and hindgut NETs $(2.3 \%)$.

\section{Percentages of distant metastases and association between MEN-1 and frequency of carcinoid syndrome in GI-NETs (Table 6)}

Among all patients with GI-NETs, distant metastases were observed at initial diagnosis in $6.0 \%$. Regarding location, midgut NETs were the most common $(9.8 \%)$ followed by foregut NETs $(8.6 \%)$ and hindgut NETs (3.5\%). With regard with the grade of WHO calcification, the percentage of distant metastases in patients with NEC at initial diagnosis was high at the rate of $32.3 \%$ compared with that in patients with NET G1/G2 at the rate of $2.7 \%$. Especially, foregut NETs patients with NEC was the most prevalent at the rate of $40.9 \%$.

Meanwhile, complications with MEN-1 were observed in $0.7 \%$ of all GI-NETs. Regarding location, they were observed in $0.7,0$, and $0.2 \%$ of foregut, midgut, and

Table 5 The trends of epidemiology of gastrointestinal neuroendocrine tumors (GI-NETs) from 2005 to 2010 in Japan

\begin{tabular}{|c|c|c|}
\hline & $2005^{*}$ & 2010 \\
\hline Total number of patients treated for GI-NETs & $4,406(95 \%$ CI $3,321-5,420)$ & $8,088(95 \%$ CI $5,669-10,507)$ \\
\hline Foregut & $1,338(95 \%$ CI $1,009-1,640)$ & $2,107(95 \%$ CI $1,189-3,028)$ \\
\hline Midgut & $423(95 \%$ CI 319-520) & $290(95 \%$ CI 271-349) \\
\hline Hindgut & 2,645 (95\% CI 1,994-3,254) & $5,690(95 \%$ CI 3,583-7,797) \\
\hline Overall prevalence of GI-NETs (per 100,000 population) & 3.45 (95\% CI 1.93-4.24) & $6.42(95 \%$ CI $4.50-8.34)$ \\
\hline Foregut & 1.05 (95\% CI 0.59-1.28) & $1.67(95 \%$ CI $0.94-2.40)$ \\
\hline Midgut & $0.33(95 \%$ CI $0.18-0.41)$ & $0.23(95 \%$ CI $0.18-0.28)$ \\
\hline Hindgut & 2.07 (95\% CI 1.56-2.55) & $4.52(95 \%$ CI 3.17-5.87) \\
\hline Incidence rate of GI-NETs (per 100,000 population) & $2.10(95 \%$ CI $1.56-2.54)$ & $3.51(95 \%$ CI $2.50-4.53)$ \\
\hline Foregut & $0.64(95 \%$ CI $0.48-0.77)$ & $1.20(95 \%$ CI $0.48-1.91)$ \\
\hline Midgut & 0.20 (95 \% CI 0.15-0.24) & 0.15 (95 \% CI 0.12-0.18) \\
\hline Hindgut & $1.26(95 \%$ CI $0.94-1.52)$ & $2.12(95 \%$ CI $1.56-2.67)$ \\
\hline
\end{tabular}

*Data modified from reference [5]

$95 \%$ CI $95 \%$ confidence interval; Foregut esophagus, stomach and duodenum; Midgut jejunum, ileum and vermiform appendix; Hindgut large intestine and colon 
Table 6 Percentages of distant metastases, associated MEN-1 and carcinoid syndrome in gastrointestinal neuroendocrine tumors (GINETs) in 2010

\begin{tabular}{|c|c|c|c|c|c|}
\hline & \multicolumn{3}{|c|}{ Distant metastases (\%) } & \multirow{2}{*}{$\begin{array}{l}\text { Associated } \\
\text { MEN-1 (\%) }\end{array}$} & \multirow{2}{*}{$\begin{array}{l}\text { Carcinoid } \\
\text { syndrome } \\
(\%)\end{array}$} \\
\hline & Total & $\begin{array}{l}\text { NET } \\
\text { G1/G2 }\end{array}$ & NEC & & \\
\hline Total GI-NETs & 6.0 & 2.7 & 32.3 & 0.42 & 3.2 \\
\hline Foregut & 8.6 & 1.8 & 40.9 & 0.72 & 1.1 \\
\hline Midgut & 9.8 & 5.9 & 28.6 & 0 & 17.1 \\
\hline Hindgut & 3.5 & 2.2 & 26.7 & 0.16 & 4.2 \\
\hline
\end{tabular}

$M E N-1$ multiple endocrine neoplasia type 1

hindgut NETs, respectively; this indicates complications with MEN-1 in GI-NETs are rare in Japan.

In addition, the frequency of carcinoid syndrome in patients with GI-NETs was $3.2 \%$. Thus, carcinoid syndrome in GI-NETs is observed less frequently in Japan than Western nations. Regarding location, midgut NETs were the most common (17.1\%) followed by foregut NETs $(4.2 \%)$ and hindgut NETs (1.1\%).

\section{Discussion}

The second nationwide epidemiological survey of patients with GEP-NETs was conducted in Japan in 2010, and the data were compared with those from 2005 to elucidate epidemiological changes.

An estimated 3,379 patients received treatment for PNETs from January 1 to December 31, 2010 in Japan; therefore, the prevalence of PNETs is about 2.69 per 100,000 people. In 2005 , these figures were 2,845 and 2.23 per 100,000 people, respectively, indicating an approximately 1.2 -fold increase in the number of patients. The incidences of new-onset PNETs in 2005 and 2010 were about 1.01 and 1.27 per 100,000 people, respectively, indicating a 5-year increase in the incidence of new-onset PNETs. Interestingly, the percentage of NFPNETs increased from $42.8 \%$ in 2005 to $65.5 \%$ in 2010 , approaching that of Western nations $[2,7,8]$. There are 2 possible reasons for this. First, the disease concept of NETs disseminated among general clinicians; that is, clinicians have become accustomed to keeping PNETs in mind when treating pancreatic tumors. Second, the availability of endoscopic ultrasonography (EUS), which is useful for the diagnosis of pancreatic diseases [9, 10], has made endoscopic ultrasound-guided fine-needle aspiration (EUS-FNA) easy to perform for pancreatic tumors, which were merely being followed-up before; thus, the pathological diagnosis of PNETs has become more accurate $[11,12]$.
An estimated 8,088 people received treatment for GINETs in Japan in 2010, which means the prevalence of patients with this disease was about 6.42 per 100,000 people; in 2005, these figures were 4,406 and 3.45 per 100,000 people, respectively, indicating a 1.8 -fold increase in the number of patients with this disease. In addition, the incidence rate of new-onset GI-NETs increased from about 2.1 per 100,000 people in 2005 to about 3.51 per 100,000 people in 2010.

Similar to the 2005 survey, few patients had midgut NETs and the locations of GI-NETs varied: 26.1, 3.6, and $70.3 \%$ in the foregut, midgut, and hindgut, respectively. In Western nations, 30-60\% of GEP-NETs are derived from midgut $[2,13,14]$ in contrast to the Japanese data. The epidemiology of GEP-NETs was recently reported in Asian nations including Taiwan [15], China [16], and Korea [17, 18]. Interestingly, the prevalence of patients with midgut NETs in these nations is low like Japan, indicating ethnic differences between Asians and Western populations.

The present study involved a survey conducted according to the 2010 WHO classification [6]. The 2010 WHO classification distinguishes between well-differentiated NETs and poorly differentiated NECs of small- or largecell type. NETs are further divided with respect to Ki-67 index: NET G1 and NET G2. Before the present survey was conducted, the frequency of NEC among GEP-NETs in Japan was not clear. A Korean study [17] reports that the frequency of NECs among all GEP-NETs is $2.84 \%$. Meanwhile, in the present survey, the frequency of NEC among all GEP-NETs in Japan was $6.7 \%(225 / 3,366)$. Interestingly, the frequency of NEC in NF-PNETs was $9.7 \%$, which is substantially higher than that reported in Western nations, where NEC in NF-PNETs is uncommon [8]. However, with regard to the grade of WHO calcification, the percentage of distant metastases in patients with NEC at initial diagnosis was high compared with that in patients with NET G1/G2. Especially, NF-PNETs patients with NEC was the most prevalent at the rate of $51.9 \%$. On the other hand, the frequency of NEC among all GI-NETs was $6.2 \%$ in the present study; the common types were foregut NEC (12.6\%), midgut NETs (9.1\%), and hindgut NETs $(2.3 \%)$. Similarilly, the percentage of distant metastases in patients with NEC at initial diagnosis was high compared with that in patients with NET G1/G2.

According to the US SEER study, distant metastases are present in $64 \%$ of PNETs followed by cecal, colonic, and small-intestinal NETs in 44, 32, and $30 \%$ of PNETs, respectively [6]. In European and American referral centers, up to 77 and $91 \%$ of patients with PNETs and intestinal NETs [19-22] present with distant metastases at initial diagnosis, respectively [13]. In the present Japanese study, patients in whom distant metastases were observed at initial diagnosis accounted for $19.9 \%$ of PNETs and 
$6.0 \%$ of GI-NETs. Regarding the location of GI-NETs, midgut NETs were the most common $(9.8 \%$ ) followed by foregut NETs $(9.8 \%)$ and hindgut NETs (3.5\%); however, these frequencies are substantially lower than those reported in Western nations. Furthermore, as shown in Table 6, the frequency of carcinoid syndrome in patients with GI-NETs is low (3.2\%) compared to that reported in Western nations, suggesting ethnic differences.

At present, 4 genetic diseases-MEN-1, von HippelLindau (VHL) disease, von Recklinghausen disease, and tuberous sclerosis - are thought to be associated with NETs [23]. As for PNETs complicated with MEN-1 [24, 25] or VHL, [26], screening must be performed at the initial diagnosis of PNETs because of different surveillance methods and treatment guidelines. MEN-1 is reported to be complicated with NF-PNETs, gastrinoma, and insulinoma at frequencies of about $80 \%, 50 \%$, and $20 \%$, respectively [23]. On the other hand, 20-25\% of gastrinomas and 4-5\% of insulinomas are reported to be complicated with MEN-1 [27]. The rate of MEN-1 association in functional PNETS in the present study (4.9\%) does not differ from that reported in Western nations $[27,28]$. However, MEN1 associated with NF-PNETs was observed in only $4.0 \%$ of cases in Japan. Furthermore, the presence of MEN-1 in GI-NETs in the present study was only $0.7 \%$, whereas approximately $30 \%$ of NF-PETs are reported to be associated with MEN-1 in Western nations [28]. The difference in the frequencies of MEN-1 in NF-PETs and GI-NETs between Japan and Western nations may be due to ethnic differences as well.

There is currently no consensus regarding antitumor chemotherapy drugs against advanced GEP-NETs in Japan, and most treatment regimens are not covered by insurance. Global clinical studies on various molecularly targeted drugs against GEP-NETs were recently conducted. The results show everolimus [29, 30], an mTOR inhibitor, and sunitinib [31, 32], a multikinase inhibitor, are effective against advanced PNETs (NET G1/G2); in addition, octreotide LAR was shown to be effective against midgutderived, metastatic, well-differentiated NETs in 2009 (PROMID study) [33]. These drugs have become reimbursable as antitumor drugs for treating advanced GI-NETs in Japan. Regarding NET, functionality, invasion depth, and the presence or absence of metastases must be correctly evaluated and treatment administered on the basis of the degrees of differentiation and malignancy of the tumor [4, 34-36]. Although surgical total excision is the standard treatment [37], some studies report that when radical treatment is difficult, debulking surgery of primary lesions and liver metastatic lesions effectively alleviate symptoms and improve prognosis [4, 34, 37]. On the other hand, in cases of unresectable advanced tumors, treatment aiming to improve prognosis by inhibiting tumor growth and improving clinical symptoms is necessary [8, 13, 27]. For this purpose, it is important to understand patient backgrounds, particularly epidemiological background, and be aware of the epidemiological differences between Japanese and Western populations. Thus, the results of the present epidemiological survey investigating the 5 -year changes in GEP-NETs in Japan will be invaluable to clinicians.

Acknowledgments This study was supported in part by Grants-inAid from the National Cancer Center Research and Development Fund (Grant Number: 21-8-6). The authors are very grateful to the doctors who responded to the questionnaires.

Conflict of interest The authors declare that they have no conflict of interest.

\section{References}

1. Metz DC, Jensen RT. Gastrointestinal neuroendocrine tumors. Gastroenterology. 2008;135:1469-92.

2. Yao JC, Hassan M, Phan A, et al. One hundred years after "carcinoid": epidemiology of and prognostic factors for neuroendocrine tumors in 35,825 cases in the United States. J Clin Oncol. 2008;26:3063-72.

3. Modlin IM, Oberg K, Chung DC, et al. Gastroenteropancreatic neuroendocrine tumours. Lancet Oncol. 2008;9:61-72.

4. Ito T, Igarashi H, Jensen RT. Therapy of metastatic pancreatic neuroendocrine tumors (pNETs): recent insights and advances. J Gastroenterol. 2012;47:941-60.

5. Ito T, Sasano H, Tanaka M, et al. Epidemiological study of gastroenteropancreatic neuroendocrine tumors in Japan. J Gastroenterol. 2010;45:234-43.

6. Bosman FT, Carneiro F, Hruban RH, et al. WHO World Health Organization classification of tumors and genetics of the digestive system. Lyon: IARC Press; 2010.

7. Pape UF, Böhmig M, Berndt U, et al. Survival and clinical outcome of patients with neuroendocrine tumors of the gastroenteropancreatic tract in a German referral center. Ann N Y Acad Sci. 2004;1014:222-33.

8. Falconi M, Bartsch DK, Eriksson B, et al. ENETS consensus guidelines for the management of patients with digestive neuroendocrine neoplasms of the digestive system: well-differentiated pancreatic non-functioning tumors. Neuroendocrinology. 2012;95:120-34.

9. Ishikawa $\mathrm{T}$, Itoh $\mathrm{A}$, Kawashima $\mathrm{H}$, et al. Usefulness of EUS combined with contrast-enhancement in the differential diagnosis of malignant versus benign and preoperative localization of pancreatic endocrine tumors. Gastrointest Endosc. 2010;71: 951-9.

10. Itokawa F, Itoi T, Sofuni A, et al. EUS elastography combined with the strain ratio of tissue elasticity for diagnosis of solid pancreatic masses. J Gastroenterol. 2011;46:843-53.

11. Haba S, Yamao K, Bhatia V, et al. Diagnostic ability and factors affecting accuracy of endoscopic ultrasound-guided fine needle aspiration for pancreatic solid lesions: Japanese large single center experience. J Gastroenterol. 2013;48:973-81.

12. Hosoda W, Takagi T, Mizuno N, et al. Diagnostic approach to pancreatic tumors with the specimens of endoscopic ultrasoundguided fine needle aspiration. Pathol Int. 2010;60:358-64.

13. Pavel M, Baudin E, Couvelard A, et al. ENETS consensus guidelines for the management of patients with liver and other distant metastases from neuroendocrine neoplasms of foregut, 
midgut, hindgut, and unknown primary. Neuroendocrinology. 2012;95:157-76.

14. Oberg K. Diagnosis and treatment of carcinoid tumors. Expert Rev Anticancer Ther. 2003;3:863-77.

15. Tsai HJ, Wu CC, Tsai CR, et al. The epidemiology of neuroendocrine tumors in Taiwan: a nation-wide cancer registry-based study. PLoS One. 2013;22(8):e62487.

16. Wang YH, Lin Y, Xue L, et al. Relationship between clinical characteristics and survival of gastroenteropancreatic neuroendocrine neoplasms: a single-institution analysis (1995-2012) in South China. BMC Endocr Disord. 2012;29(12):30. doi:10.1186/ 1472-6823-12-30.

17. Cho MY, Kim JM, Sohn JH, et al. Current trends of the incidence and pathological diagnosis of gastroenteropancreatic neuroendocrine tumors (GEP-NETs) in Korea 2000-2009: multicenter study. Cancer Res Treat. 2012;44:157-65.

18. Lim T, Lee J, Kim JJ, et al. Gastroenteropancreatic neuroendocrine tumors: incidence and treatment outcome in a single institution in Korea. Asia Pac J Clin Oncol. 2011;7:293-9.

19. Pape UF, Berndt U, Müller-Nordhorn J, et al. Prognostic factors of long-term outcome in gastroenteropancreatic neuroendocrine tumours. Endocr Relat Cancer. 2008;15(4):1083-97.

20. Ekeblad S, Skogseid B, Dunder K, et al. Prognostic factors and survival in 324 patients with pancreatic endocrine tumor treated at a single institution. Clin Cancer Res. 2008;14(23):7798-803.

21. Strosberg J, Gardner N, Kvols L. Survival and prognostic factor analysis of 146 metastatic neuroendocrine tumors of the mid-gut. Neuroendocrinology. 2009;89(4):471-6.

22. Ahmed A, Turner G, King B, et al. Midgut neuroendocrine tumours with liver metastases: results of the UKINETS study. Endocr Relat Cancer. 2009;16(3):885-94.

23. Alexakis N, Connor S, Ghaneh P, et al. Hereditary pancreatic endocrine tumours. Pancreatology. 2004;4(5):417-33.

24. Ito $\mathrm{T}$, Igarashi $\mathrm{H}$, Uehara $\mathrm{H}$, et al. Causes of death and prognostic factors in multiple endocrine neoplasia type 1: a prospective study: comparison of 106 MEN1/Zollinger-Ellison syndrome patients with 1613 literature MEN1 patients with or without pancreatic endocrine tumors. Medicine (Baltimore). 2013;92(3):135-81.

25. Niina Y, Fujimori N, Nakamura T, et al. The current strategy for managing pancreatic neuroendocrine tumors in multiple endocrine neoplasia type 1. Gut Liver. 2012;6(3):287-94.

26. Igarashi $\mathrm{H}$, Ito $\mathrm{T}$, Nishimori I, et al. Pancreatic involvement in Japanese patients with von Hippel-Lindau disease: results of a nationwide survey. J Gastroenterol. 2013. (Epub ahead of print). PMID 23543325.

27. Jensen RT, Cadiot G, Brandi ML, et al. ENETS consensus guidelines for the management of patients with digestive neuroendocrine neoplasms: functional pancreatic endocrine tumor syndromes. Neuroendocrinology. 2012;95(2):98-119.

28. Oberg K, Eriksson B. Endocrine tumours of the pancreas. Best Pract Res Clin Gastroenterol. 2005;19:753-81.

29. Yao JC, Shah MH, Ito T, et al. Everolimus for advanced pancreatic neuroendocrine tumors. $\mathrm{N}$ Engl $\mathrm{J}$ Med. 2011;364(6):514-23.

30. Ito T, Okusaka T, Ikeda M, et al. Everolimus for advanced pancreatic neuroendocrine tumours: a subgroup analysis evaluating Japanese patients in the RADIANT-3 trial. Jpn J Clin Oncol. 2012;42(10):903-11.

31. Raymond E, Dahan L, Raoul JL, et al. Sunitinib malate for the treatment of pancreatic neuroendocrine tumors. $\mathrm{N}$ Engl J Med. 2011;364(11):1082.

32. Ito T, Okusaka T, Nishida T, et al. Phase II study of sunitinib in Japanese patients with unresectable or metastatic, well-differentiated pancreatic neuroendocrine tumor. Invest New Drugs. 2013;31:1265-74.

33. Rinke A, Müller HH, Schade-Brittinger C, et al. Placebo-controlled, double-blind, prospective, randomized study on the effect of octreotide LAR in the control of tumor growth in patients with metastatic neuroendocrine midgut tumors: a report from the PROMID study group. J Clin Oncol. 2009;27(28):4656-63.

34. Ito T, Igarashi H, Jensen RT. Pancreatic neuroendocrine tumors: clinical features, diagnosis and medical treatment: advances. Best Pract Res Clin Gastroenterol. 2012;26(6):737-53.

35. Tsutsumi K, Ohtsuka T, Mori Y, et al. Analysis of lymph node metastasis in pancreatic neuroendocrine tumors (PNETs) based on the tumor size and hormonal production. J Gastroenterol. 2012;47(6):678-85.

36. Ito T, Tanaka M, Sasano H, et al. Preliminary results of a Japanese nationwide survey of neuroendocrine gastrointestinal tumors. J Gastroenterol. 2007;42(6):497-500.

37. Imamura $M$. Recent standardization of treatment strategy for pancreatic neuroendocrine tumors. World J Gastroenterol. 2010;16(36):4519-25. 